\title{
Prediction of motor function by diffusion tensor tractography in patients with basal ganglion haemorrhage
}

\author{
Jingsong Zeng, Ping Zheng, Junfa Xu, Wusong Tong, Yijun Guo, Wenjin Yang, Gaoyi Li, Bin He
}

Department of Neurosurgery, Pudong New Area People's Hospital, Shanghai, China

Submitted: 1 September 2010

Accepted: 10 December 2010

Arch Med Sci 2011; 7, 2: 310-314

DOI: 10.5114/aoms.2011.22083

Copyright ๑ 2011 Termedia \& Banach

\begin{abstract}
Introduction: Haemorrhagic stroke is one of the leading causes of death and the most common cause of long-term adult disability. An accurate estimation of prognosis is very important for haemorrhagic stroke patients. Impairment of motor function caused by pyramidal tract injury is common in these patients. In this study, we performed MR diffusion tensor tractography (DTT) to predict the impairment of motor function in patients with basal ganglion haemorrhage and explore its clinical value.

Material and methods: Diffusion tensor tractography was performed in 33 patients with basal ganglia haemorrhage within 2 weeks after onset to visualize the course of pyramidal tracts (PTs), and patients were classified into four groups according to the fibre ratio of PTs, calculated by dividing the PT number of the affected hemisphere by that of the unaffected hemisphere, as follows: type $A$, the fibre ratio was less than $1 / 4$; type $B$, less than $1 / 2$; type $C$, more than $1 / 2$; and type $D$, more than $3 / 4$. The upper extremity motricity index (UMI) was used to evaluate the motor function at onset and 6 months after onset. Upper extremity motricity index scores were compared among the different groups and a Spearman analysis was performed to correlate the UMI scores with different integrity of pyramidal tracts.

Results: There were no differences in the UMI scores at onset among the 4 groups $(p>0.05)$. The UMI scores obtained at 6 months after onset were significantly unequal and were influenced by the DTT type $(p<0.05)$. There was a significant correlation between the integrity of the pyramidal tracts and the UMI scores 6 months after onset $(r=0.7312, p<0.05)$.

Conclusions: There was a positive correlation between the integrity grade of pyramidal tracts and the motor function, showing that the more seriously were the pyramidal tracts damaged, the worse was the motor function. The DTT findings of the pyramidal tract in acute cerebral haemorrhage may valuably predict the motor function outcome.
\end{abstract}

Key words: basal ganglion haemorrhage, diffusion tensor tractography, pyramidal tract, upper extremity motricity index.

\section{Introduction}

Haemorrhagic stroke has a high long-term disability rate. The commonest cause is impairment of motor function induced by pyramidal tract injury in basal ganglion haemorrhagic patients [1]. Thus an early prediction of long-term outcome of motor function is critically important in terms of rehabilitation for these patients.

\author{
Corresponding author: \\ Ping Zheng \\ Department of Neurosurgery \\ Pudong New Area \\ People's Hospital \\ 490 South Chuanhuan Road \\ Shanghai 201200, China \\ E-mail: jojo_ras@hotmail.com
}


Diffusion tensor imaging (DTI) is an MRI technique for evaluating brain structure by measuring tissue water diffusion in 3-dimensional (3-D) space [2]. Diffusion tensor tractography (DTT) noninvasively visualizes the course of major white matter tracts based on the DTI technique $[3,4]$ and can be used to detect long patterns of orientation as well as integrity of white matter tracts [5]. In addition, it provides information about the course, the compression, displacement or interruption of white matter tracts in three dimensions [6].

Previous studies have demonstrated that DTI can evaluate injury of the lateral corticospinal tract (CST) in patients with intracerebral haemorrhage [7]. These studies focused on the origin of CSTaffected hemisphere. However, to date, the role of the number of pyramidal tracts in prediction of motor outcome in haemorrhagic stroke patients has not been well addressed [8]. Here we hypothesized that DTT evidence of the degree of damage of the pyramidal tracts may reflect motor function impairment in haemorrhagic stroke patients. Therefore, in this prospective study we sought to observe the degree of impairment of pyramidal tracts assessed by DTT and relate their status to the motor function outcome.

\section{Material and methods}

\section{Patients}

From November 2006 to November 2008, 45 patients with haemorrhagic stroke confirmed by CT scan were admitted to our department. Of these, 12 cases were excluded because of missing data and 33 cases were enrolled. Inclusion criteria including DTI data were prospectively collected within 2 weeks after onset. Haemorrhagic stroke confined within the basal ganglia was confirmed by the initial head CT scan. We excluded patients with previous stroke history and history of pyramidal system injury. All of our patients were treated with conservative medical therapies. Signed informed consent documents were provided by all patients or appropriate family members.

\section{Diffusion tensor tractography}

MR imaging was performed on a 1.5T Signa Excite II (GE Signa, Milwaukee, WI, USA) with the same protocol. For DTI, we applied a single shot spin echo diffusion-weighted echo planar imaging (DW-EPI) sequence with head coil (TE $=64.8 \mathrm{~ms}$, $\mathrm{TR}=8000 \mathrm{~ms}$, matrix size $=128 \times 128, \mathrm{FOV}=240$ $\mathrm{mm}$, slice thickness $=3-5 \mathrm{~mm}, \mathrm{~b}$ values of 0 and $1000 \mathrm{~s} / \mathrm{mm}^{2}$, no intersection gap). The Volume-One and Dtv software (Tokyo University, Japan) was used to reconstruct and visualize the fibre tracts. A seed region of interest (ROI) was drawn in the PT portion of the anterior mid-pons on a 2D map and another ROI was drawn in the PT portion of the anterior lower pons on a 2D map (Figure 1). The fibre tracts passing through both ROI were designated as the final tracts of interest. Diffusion tensor tractography was performed by an examiner blinded to the patient's motor function evaluation. Before tracking was initiated, the user could adjust the FA threshold and the minimum fibre length (stop criteria). Our default FA threshold was 0.18. Other parameters were set as default according to a previous study on the optimal tractability threshold of FA [9].

The examiner blinded to the patients calculated PT numbers by using Dtv software which has a target function that calculates fibre number (tracked lines) automatically along the selected portion of the constructed tract. For the integrity of pyramidal tracts, the DTT data were classified into four groups according to the fibre ratio, as calculated by dividing the PT number of the affected hemisphere by that of the unaffected hemisphere, as follows: type $A$, the fibre ratio was less than $1 / 4$; type $B$, less than $1 / 2$; type $C$, more than $1 / 2$; and type D, more than 3/4 [7].

\section{Motor function assessments}

All patients underwent UMI examinations at onset and six months after onset by an experienced neurosurgeon blinded to the DTT data. All patients were subject to the standard physical and poststroke occupational therapy. The UMI is frequently used to measure motor function with a score range of 0-100 [10]. Higher scores reflect more complete functional recovery [10].

\section{Statistical analysis}

One-way ANOVA test, a non-parametric statistical analysis tool from SPSS 12.0 software (SPSS Inc, Chicago, USA), was employed due to the small and different numbers of the four DTT type groups. To determine the relationship between motor function and the status of pyramidal tract

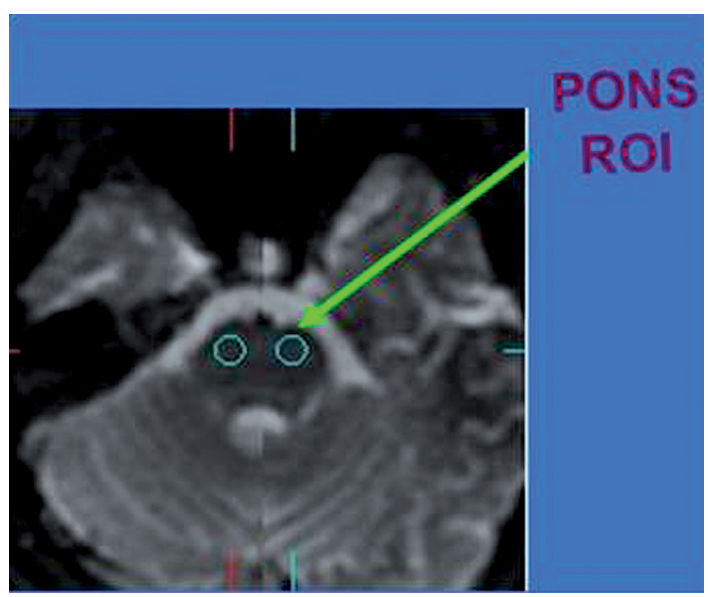

Figure 1. Region of interest in the pons: DTI imaging 
injury, we performed a Spearman analysis of UMI scores against the degree of damage of the pyramidal tract. A threshold of $p<0.05$ was used.

\section{Results}

The courses of pyramidal tracts were successfully identified in all 33 subjects with our methods. This study included 24 male and 9 female patients with an average age of 60 (range 48-75) years. Distribution of "age", "gender" and "haematoma size" of the 4 DTT type groups were not significantly different among the groups ( $p>0.05$ ).

The study population was divided into 4 types by the fibre ratio: 4 type $A, 11$ type $B, 12$ type $C$ and 6 type D. Figures 2-5 were taken from four typical patients with DTT type A, B, C and D, respectively.

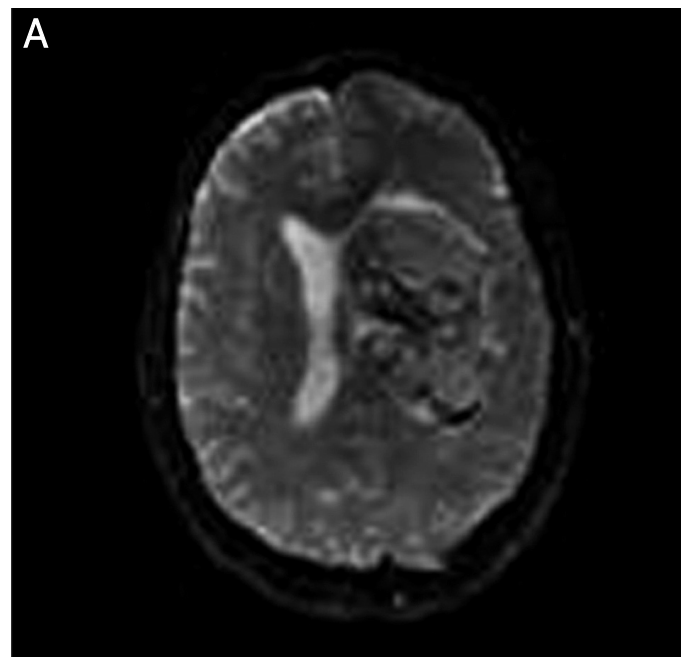

There were no differences in the UMI scores at onset among the four groups $(p>0.05)$. The UMI scores obtained at 6 months after onset were significantly unequal and were influenced by the DTT type $(p<0.05)$. The average scores at 6 months after onset were smallest in the DTT type A $(46.38 \pm 15.64)$ and greatest in the type $D$ group $(84.00 \pm 12.02)$ (Table I). We found that the integrity of the pyramidal tract was positively correlated with the UMI scores at 6 months after onset $(r=0.7312$, $p<0.05)$.

\section{Discussion}

We performed a prospective study to evaluate the integrity of pyramidal tracts classified by DTT type during the early stage of haemorrhagic stroke and

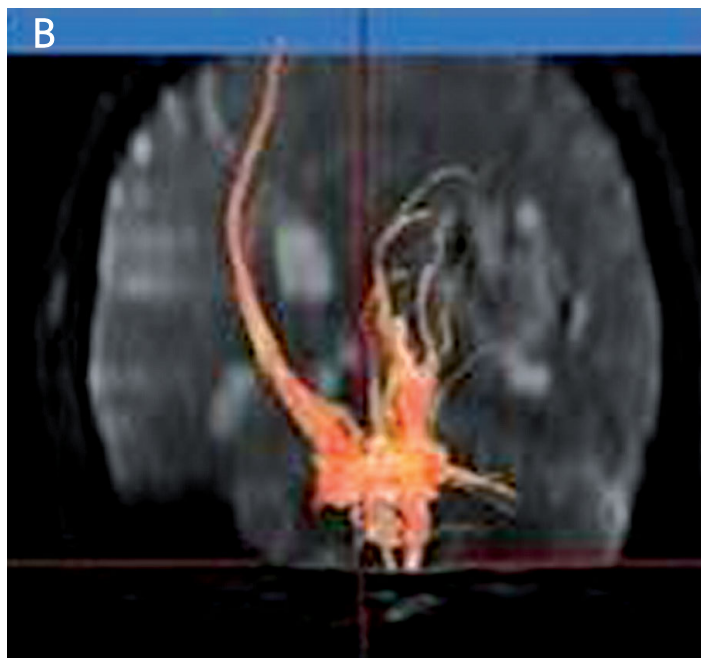

Figure 2. Male, 56 years old: A - original DTI picture obtained $8 \mathrm{~h}$ after onset showed left basal ganglion haematoma; B - fibre tracking showed the haematoma interrupting the pyramidal tract (group A) UMI score: 46 (fibre ratio was less than $1 / 4$ )
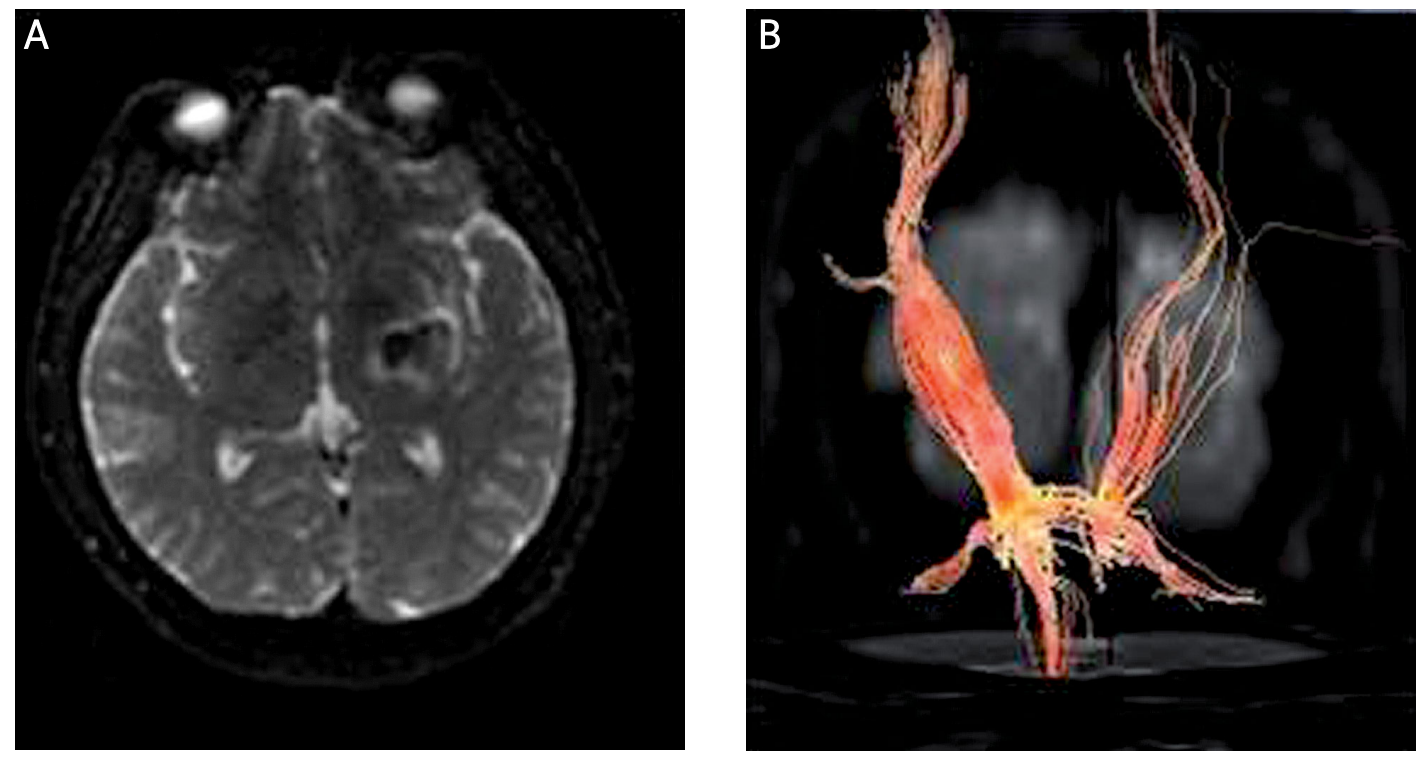

Figure 3. Male, 61 years old: A - original DTI picture obtained $36 \mathrm{~h}$ after onset showed left basal ganglion haematoma; B - fibre tracking showed the haematoma occupying the pyramidal tract (group B) UMI score: 70 (fibre ratio was less than $1 / 2$ ) 

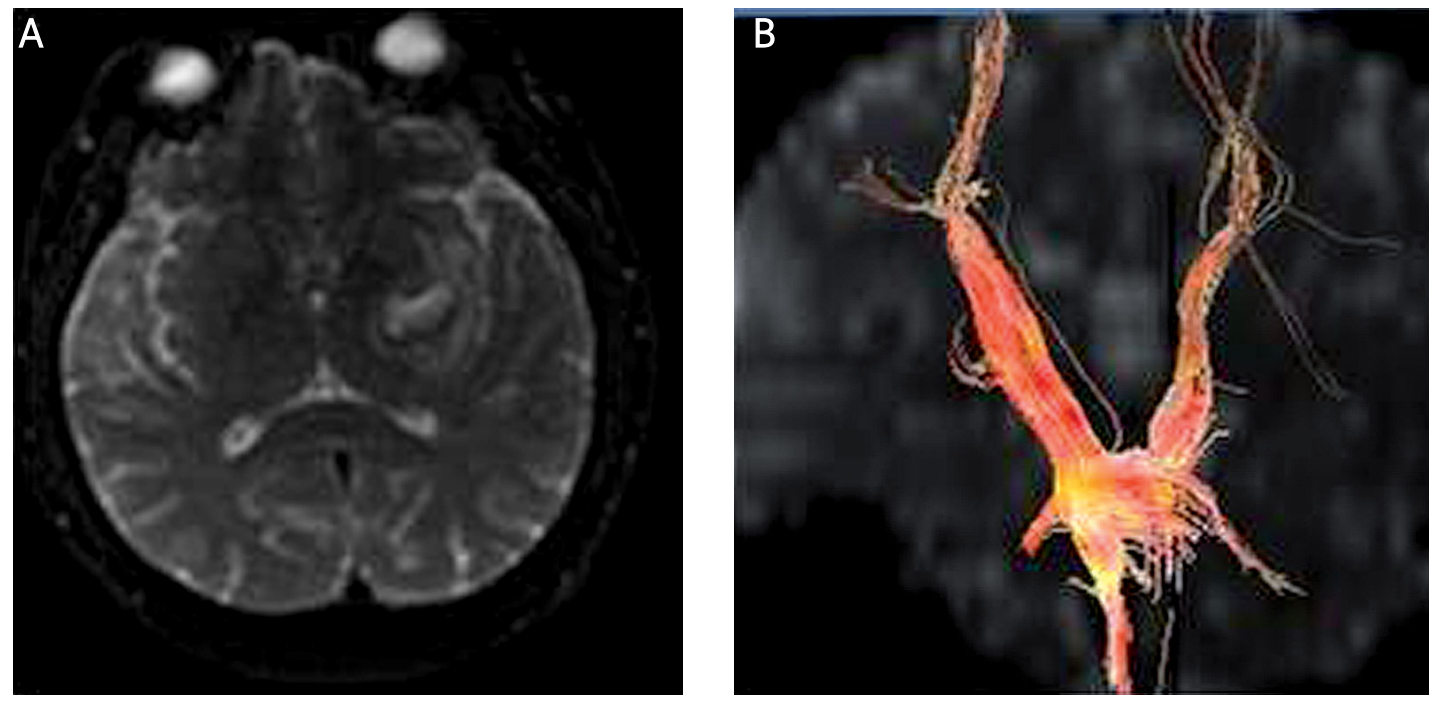

Figure 4. Male, 55 years old: A - original DTI picture obtained $15 \mathrm{~h}$ after onset showed left basal ganglion haematoma; B - fibre tracking showed the haematoma displacing the pyramidal tract (group C) UMI score: 88 (fibre ratio was more than $1 / 2$ )
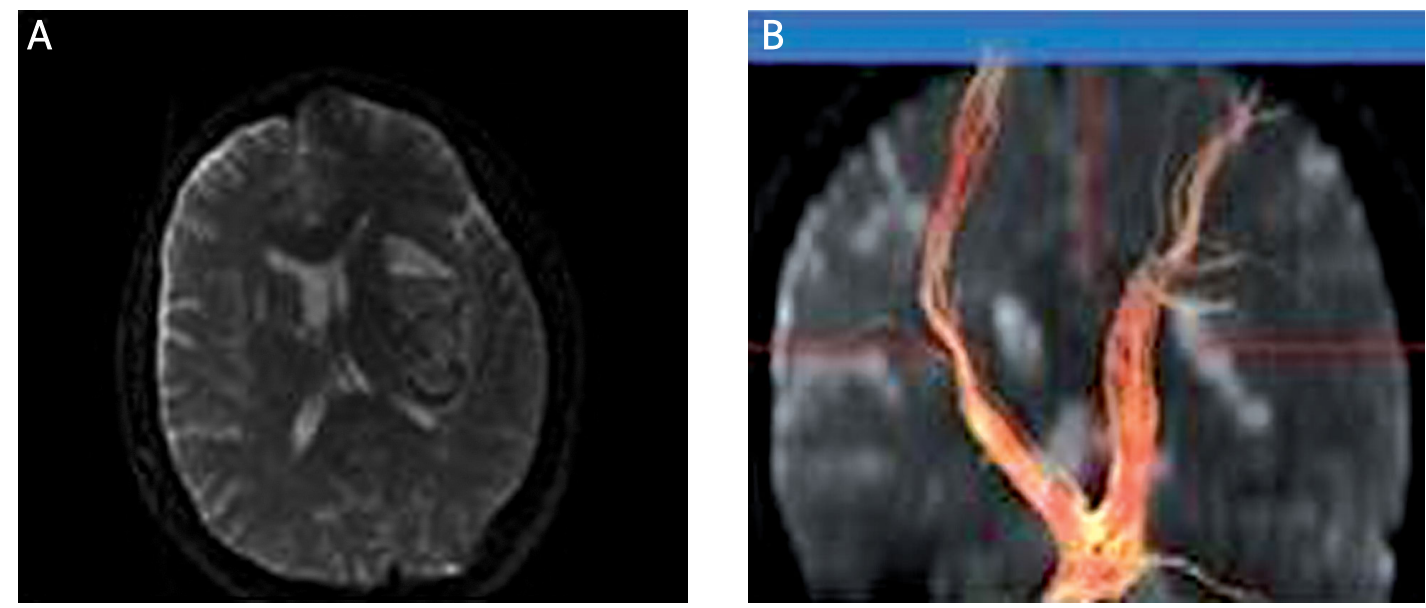

Figure 5. Female, 51 years old: A - original DTI picture obtained $5 \mathrm{~h}$ after onset showed left basal ganglion haematoma;. B - fibre tracking showed the haematoma compressing the pyramidal tract (group D) UMI score: 90 (fibre ratio was more than 3/4)

Table I. Upper extremity scores distributed among different DTT types

\begin{tabular}{|lcccccc|}
\hline Group & $\begin{array}{c}\text { Case } \\
\text { number }\end{array}$ & $\begin{array}{c}\text { Fibre number } \\
\text { in affected } \\
\text { hemisphere }\end{array}$ & $\begin{array}{c}\text { Fibre number } \\
\text { in unaffected } \\
\text { hemisphere }\end{array}$ & $\begin{array}{c}\text { Fibre ratio } \\
\text { group }\end{array}$ & & \multicolumn{2}{c|}{ UMl scores } \\
\cline { 6 - 7 } A & 4 & $41 \pm 15$ & $208 \pm 21$ & $<1 / 4$ & $8.61 \pm 5.07^{*}$ & $\begin{array}{c}\text { Initial } \\
\text { after onset }\end{array}$ \\
\hline B & 11 & $87 \pm 14$ & $226 \pm 18$ & $<1 / 2$ & $8.76 \pm 4.93^{*}$ & $68.38 \pm 15.64$ \\
\hline C & 12 & $124 \pm 22$ & $219 \pm 29$ & $>1 / 2$ & $9.70 \pm 6.96^{*}$ & $78.81 \pm 12.89^{\#}$ \\
\hline D & 6 & $198 \pm 18$ & $232 \pm 23$ & $>3 / 4$ & $10.72 \pm 9.89^{*}$ & $84.00 \pm 12.02^{\#}$ \\
\hline
\end{tabular}

No significant difference was found among the four groups, ${ }^{*} p>0.05$, compared with type $A,{ }^{*} p<0.05$

found that it correlated with the motor outcome. We defined the fibre ratio between the affected and unaffected hemisphere as the degree of damage of the pyramidal tract. Moreover, our results suggested that DTT measures were valid structural surrogates of motor impairment after stroke.
Our quantitative analysis of 33 patients with varying degrees of motor recovery revealed a highly significant relationship between DTT-derived parameters and motor impairment scores. These results support previous studies, which associate the pattern of pyramidal tract damage with motor 
impairment using qualitative DTI approaches [7, 11]. In another study, Yoshioka et al. [7, 12] reported that motor functional outcome in patients with intracerebral haemorrhage can be predicted by measuring FA values using diffusion tensor tractography. They thought that the decrease in FA values in the group with poor motor functional outcome likely resulted from damage to neural fibres by the haematoma in the acute phase. In our opinion, we have added direct viewing evidence to their study.

The scoring tool of UMI score was chosen to focus on the clinical perspective of the functional evaluation. At the initial evaluation, none of the patients could move his/her fingers voluntarily. The scores measured the motor recovery after 6 months as indicated in each patient. Those with greatly injured pyramidal tracts (group A and B) had worse motor function, and vice versa: patients with slightly injured PTs (group C and D) had a correspondingly better outcome. Therefore, motor recovery at 6 months after onset was significantly different among these patients according to the initial DTT findings of the damaged pyramidal tracts $(p<0.05)$. An early prediction for motor recovery has great value in stroke rehabilitation; thus the clinical application of the DTT technique will empower clinicians with a better rehabilitation strategy.

The current study has the limitation that the DTT analysed was not the PT for only the hand, although we tried to predict the motor function of the affected upper extremity. And, estimation of the number of PT using ROI methods may not be accurate because the PT can by displaced by a haematoma and/or vasogenic oedema in stroke patients. We think that further combined studies using DTT with $\mathrm{FMRI}$ and TMS are required for more accurate knowledge of the PT because DTT has several limitations such as susceptibility artefact and operator-dependent technique [13].

In conclusion, our study demonstrates that DTT can reliably depict the pyramidal tract and effectively predict the motor function defects in stroke patients. The DTT finding of the pyramidal tract in acute cerebral haemorrhage is a valid structural marker of motor impairment to predict the motor function outcome. In addition, we suggest that this DTT method can be applied to other lesions in pyramidal tracts for predicting motor outcome.

\section{References}

1. Stinear CM, Barber PA, Smale PR, et al. Functional potential in chronic stroke patients depends on corticospinal tract integrity. Brain 2007; 130: 170-80.

2. Pierpaoli C, Jezzard P, Basser PJ, et al. Diffusion tensor MR imaging of the human brain. Radiology 1996; 201: 637-48.
3. Basser PJ, Pajevic S, Pierpaoli C, et al. In vivo fiber tractography using DT-MRI data. Magn Reson Med 2000; 44: 625-32.

4. Lazar M, Alexander AL, Thottakara PJ, et al. White matter reorganization after surgical resection of brain tumors and vascular malformations. Am J Neuroradiol 2006; 27 : 1258-71.

5. Mukherjee P, Berman JI, Chung SW, et al. Diffusion tensor $M R$ imaging and fiber tractography : theoretic underpinnings. Am J Neuroradiol 2008; 29: 632-41.

6. Nguyen TH, Yoshida M, Stievenart JL, et al. MR tractography with diffusion tensor imaging in clinical routine. Neuroradiology 2005; 47: 334-43.

7. Nelles M, Gieseke J, Flacke S, et al. Diffusion tensor pyramidal tractography in patients with anterior choroidal artery infarctsAm J Neuroradiol 2008; 29: 488-93.

8. Jang SH, Kim SH, Cho SH, et al. Demonstration of motor recovery process in a patient with intracerebral hemorrhage. Neuro Rehabilitation 2007; 22: 141-5.

9. Kunimatsu A, Aoki S, Masutani Y, et al. The optimal trackability threshold of fractional anisotropy for diffusion tensor tractography of the corticospinal tract. Magn Reson Med Sci 2004; 3: 11-7.

10. Demeurisse G, Demol O, Robaye R. Motor evaluation in vascular hemiplegia. Eur Neurol 1980; 19: 382-9.

11. Konishi J, Yamada K, Kizu O, et al. MR tractography for the evaluation of functional recovery from lenticulostriate infarcts. Neurology 2005; 64: 108-13.

12. Yoshioka H, Horikoshi T, Aoki S, et al. Diffusion tensor tractography predicts motor functional outcome in patients with spontaneous intracerebral hemorrhage. Neurosurgery 2008; 62: 97-103.

13. Lee SK, Kim DI, Kim J, et al. Diffusion-tensor MR imaging and fiber tractography: a new method of decribing aberrant fiber connections in developmental CNS anomalies. Radiographics 2005; 25: 53-65. 